\title{
PROPERTIES OF STRAINS OF STAPHYLOCOCCUS AUREUS IN THE 94, 96 COMPLEX
}

\author{
Elizabeth H. Asheshov, A. W. Coe and Audrey Porthouse \\ Central Public Health Laboratory, Colindale Avenue, London NW9 $5 H T$
}

\begin{abstract}
PHAGES 94 and 96 were introduced into the basic set of phages for typing strains of Staphylococcus aureus after they were shown to be useful in identifying otherwise untypable strains of this organism (Subcommittee on phage-typing of staphylococci, 1975). Phage 94, of serological group A, was isolated in the United States in 1971 (Blouse et al., 1973), and phage 96, a serological group-B phage, in New Zealand by Mrs J. Markham about 1 year later. Although the phages were isolated at different times in different parts of the world and are serologically distinct, they tend to have a rather similar range of lytic activity, and staphylococci that are sensitive to these phages are usually resistant to other basic-set phages. Such strains account for between 9 and $10 \%$ of all isolates typed at the Staphylococcus Reference Laboratory, Colindale, at present. We have examined a number of them and find that most have, as reported here, some rather distinctive properties. This suggests that they form a homogeneous group of strains with a possible common origin.
\end{abstract}

\section{MATERIALS AND METHODS}

Strains of Staphylococcus aureus. Over 200 strains of staphylococci of the 94,96 complex were examined. They included the propagating strains (PS) for the basic-set typing phages 94 and 96 and for phages $14,15,16$ and 17 . The remainder were strains sent to this laboratory for routine phage typing and represented isolates collected during 1973 to 1975 from 40 different hospitals. All were lysed by phage 94 or phage 96 or by both, and were resistant to lysis by other basic-set phages. Strains used as indicators of lysogenicity were the PS for the 22 basic-set phages and three additional "polyvalent" strains (nos. 1030, 18042 and W57) which are sensitive to most staphylococcal phages.

Phage typing was performed according to Blair and Williams (1961). In addition to the 23 phages in the current basic set, five phages showing variable activity against strains susceptible to phages 94 and 96 were tested against a selection of strains. These were phage 47A (no. NCTC8407), which was included in the basic set until the early 1950's, and four phages-nos. 14, 15, 16 and 17-isolated in the Netherlands and kindly supplied by Dr N. van Leeuwen.

Tests for lysogenicity. Three-hour cultures in nutrient broth (Oxoid no. 2) were exposed to $0.5 \mu \mathrm{g}$ of mitomycin $\mathrm{C}$ per $\mathrm{ml}$ for $30 \mathrm{~min}$. at $37^{\circ} \mathrm{C}$. The cultures were then centrifuged, the supernates discarded, and the cells resuspended in the original volume of nutrient broth. After a further $2 \mathrm{~h}$ at $37^{\circ} \mathrm{C}$, the cultures were centrifuged and the supernates spotted on to nutrient-agar plates which had been flooded with broth cultures of indicator strains.

Mutagen treatment. Two mutagens were used-ethyl methane sulphonate (EMS) and $\mathrm{N}$-methyl-N'-nitroso-N-nitroguanidine (NG). Mutagenesis with EMS was as described by

Received 10 Aug. 1976; accepted 16 Sept. 1976.

J. MEd. MICROBIOL.-VOL. 10 (1977) 


\section{TABLE I}

Patterns of lysis by seven phages, and frequency of lysogeny, among Staphylococcus aureus strains belonging to the 94,96 complex

\begin{tabular}{|c|c|c|c|c|c|c|c|c|}
\hline \multicolumn{7}{|c|}{ Reactions* with phage } & \multicolumn{2}{|c|}{ Number of } \\
\hline $47 \mathrm{~A}$ & 94 & 96 & 14 & 15 & 16 & 17 & pattern indicated & $\begin{array}{l}\text { lysogenic } \\
\text { strains }\end{array}$ \\
\hline - & + & + & + & + & - & - & 26 & 7 \\
\hline+ & + & + & + & + & - & - & 23 & 7 \\
\hline- & + & + & + & + & + & + & 14 & 4 \\
\hline+ & + & + & + & + & + & + & 10 & 2 \\
\hline- & - & + & + & + & - & - & 7 & 6 \\
\hline- & - & + & + & + & + & + & 6 & 4 \\
\hline+ & - & + & + & + & - & - & 3 & 2 \\
\hline- & + & - & + & + & -- & - & 2 & 2 \\
\hline- & - & + & t & - & - & - & 2 & 2 \\
\hline+ & - & + & + & + & + & + & $\overline{2}$ & 2 \\
\hline- & + & + & + & - & - & - & 1 & $\overline{1}$ \\
\hline+ & + & + & + & - & - & - & 1 & 1 \\
\hline - & + & + & - & + & + & + & 1 & 1 \\
\hline+ & - & + & - & - & + & + & 1 & 1 \\
\hline+ & - & - & + & + & - & - & 1 & 1 \\
\hline \multicolumn{7}{|c|}{ Any pattern } & 100 & 43 \\
\hline
\end{tabular}

$*=>50$ Plaques; $-=$ no reaction.

Novick (1963). Mutagenesis by NG was as follows: an overnight broth culture of the strain was diluted 1 in 20 in nutrient broth containing $50 \mu \mathrm{g}$ of NG per $\mathrm{ml}$ and incubated at $37^{\circ} \mathrm{C}$ for $1.5 \mathrm{~h}$. The bacteria were sedimented, washed once, resuspended in nutrient broth and incubated at $37^{\circ} \mathrm{C}$ for $18 \mathrm{~h}$ before screening for mutants.

Biological properties of strains of $S$. aureus were determined as follows: haemolysins $\alpha, \beta$ and $\delta$ by the method of Elek and Levy (1950); fibrinolysin by the method of Lack (1956); lipase on Tween 80 agar (Sierra, 1957); pigment production on cream agar (Willis, Smith and O'Connor, 1960); enterotoxin production as described by Simkovičová and Gilbert (1971).

Drug-resistance tests were performed on Oxoid no. 2 Agar. For disk-diffusion tests, the inoculum was diluted to give semi-confluent growth, and antibiotic contents per disk were: benzylpenicillin 1 unit, tetracycline $10 \mu \mathrm{g}$, streptomycin $10 \mu \mathrm{g}$, chloramphenicol $10 \mu \mathrm{g}$, erythromycin $10 \mu \mathrm{g}$, oleandomycin $5 \mu \mathrm{g}$, lincomycin $2 \mu \mathrm{g}$, neomycin $10 \mu \mathrm{g}$, bacitracin 8 units, novobiocin $5 \mu \mathrm{g}$, gentamicin $10 \mu \mathrm{g}$ and trimethoprin $2.5 \mu \mathrm{g}$; zone sizes were measured after incubation overnight at $37^{\circ} \mathrm{C}$. Resistance to methicillin was tested by making parallel streaks of undiluted overnight broth culture across a plate, and applying a strip $(121 \mathrm{~mm} \times$ $7 \mathrm{~mm}$ ) of Ford's Blotting Paper (110 $\mu \mathrm{g}$ of methicillin per strip) at right-angles to the streaks; the extent of inhibition was measured after incubation overnight at $30^{\circ} \mathrm{C}$.

\section{RESULTS}

\section{Distribution of typing patterns of strains in the 94, 96 complex}

One hundred consecutively received strains that were sensitive either to phage 94 or phage 96 , or to both phages, were examined for lysis by the five additional phages that are active against such strains. Fifteen different patterns 
were observed (table I), but five of these patterns accounted for $80 \%$ of the strains. Most strains were lysed by phages $94,96,14$ and 15, somewhat less than half were sensitive to phage $47 \mathrm{~A}$, and only 15 strains were lysed by phages 16 and 17. In general, phages 96,14 and 15 showed a similar range of activity, as did phages 16 and 17 . The four most useful phages for identifying different lytic patterns were phages $47 \mathrm{~A}, 94,96$ and either 16 or 17 . With these four phages, 10 distinct patterns could be recognised, and those that were not distinguished were the less common ones. In certain types of epidemiological investigation, the two extra phages might be useful for making fine subdivisions within the 94 , 96 complex.

\section{Lysogenicity of strains}

It seemed possible that resistance of strains in the 94, 96 complex to lysis by other basic-set phages might be due to specific immunity imposed by a particular prophage or prophages. Eighteen strains which were epidemiologically distinct and representative of different typing patterns within the complex were examined for lysogenicity with the basic-set PS and nos. 1030, 18042 and W57 as indicators. Eight strains were demonstrably lysogenic when their supernates were tested against PS94 and PS96, but showed weak and variable lysis on one or other of the polyvalent strains and failed to lyse any of the other propagating strains. The phages carried by these eight strains were purified by these single-plaque isolations on PS96. Each was then used to lysogenise PS96 and the effect of lysogenisation on the typing pattern examined. Three phages failed to change the typing pattern; one phage blocked sensitivity to phages $47 \mathrm{~A}$ and 96 ; one blocked sensitivity to phage 94 ; one blocked sensitivity to phage $47 \mathrm{~A}$; two blocked sensitivity to phages $47 \mathrm{~A}$ and 94 .

The 100 strains of $S$. aureus were then examined for lysogenicity, but with PS96 only as indicator, because the previous experiment had indicated that this would detect all or most of the carried phages. Forty-three strains were frankly lysogenic (table I). Lysogenicity was found most often in strains with the less common typing patterns and was closely associated with resistance to lysis by phages $94,96,14$ or 15 . It would seem, therefore, that differences in typing patterns within the complex may be explained by specific prophage immunity. However, the fact that most strains in the complex were not apparently lysogenic argues against prophage immunity as a major factor in resistance to lysis by other basic-set phages. Moreover, the narrow host range of the phages released by lysogenic strains in the complex suggests that these strains possess a strict restriction and modification (RM) system, and that this is responsible for their insusceptibility to the other typing phages.

Restriction and modification by PS96. Although PS96 is resistant to lysis by other basic-set phages when these are tested at the highest concentration usually employed in phage typing $(\mathrm{RTD} \times 100$, i.e., 100 times the strength of the routine test dilution), several of the phages produced a few plaques on PS96 when they were applied at a higher concentration $(\mathrm{RTD} \times 10000)$. Plaques produced by phages $80,6,47,75$ and 81 on lawns of PS96 were picked and the 


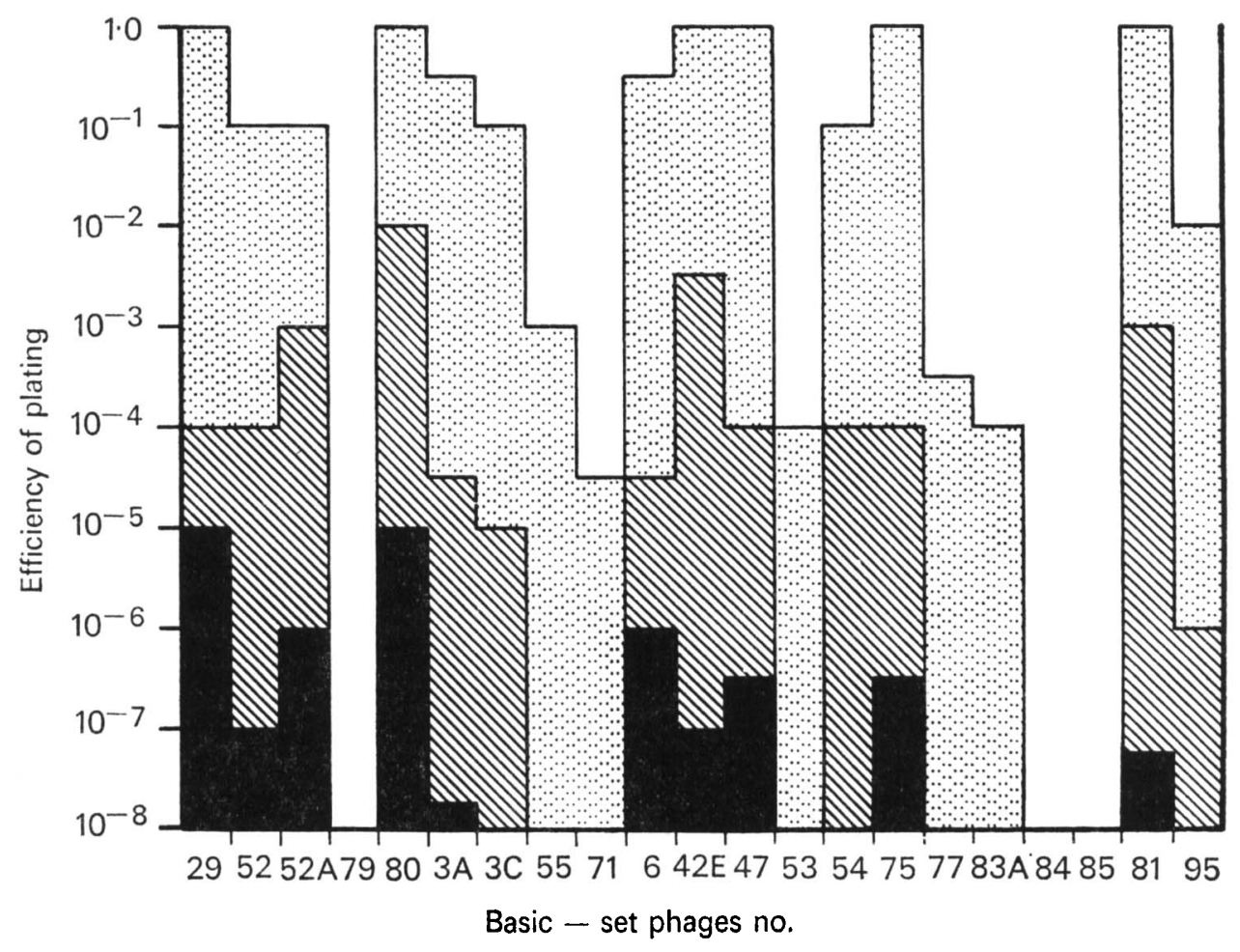

FIGURE-Efficiency of plating of 21 basic-set phages on PS96 (solid bars), on the single-step mutant $\mathrm{R}^{-}{ }_{5}$ (hatched bars) and on the double mutant $\mathrm{R}^{-}{ }_{5.16}$ (stippled bars).

phages propagated on PS96 to high titre. The lytic spectra of these phages were markedly changed, in that the phages now lysed strains in the 94,96 complex to titre but had a greatly reduced efficiency of plating (e.o.p.) on their original propagating strains. Phage 80 became almost indistinguishable in its host range from phage 96; phage 47 became identical in host range with phage $47 \mathrm{~A}$, and phages 6,75 and 81 acquired host ranges similar to that of phage 94 . PS96 therefore appears to have a RM system that prevents phages with a different host specificity from replicating, and modifies phages that escape restriction to host specificity for $94,96-$ complex strains.

Isolation of restrictionless $\left(R^{-}\right)$mutants in PS96. The strict RM system found in PS96 seemed to provide a useful means of investigating the genetics of restriction and modification in S. aureus. Attempts were first made to isolate restrictionless $\left(\mathrm{R}^{-}\right)$mutants from PS96. The method used to select such mutants relied on the assumption that loss of restriction should result in an increase in sensitivity to some of the basic-set phages that previous experiments had shown were able to grow and be modified in PS96. Phage 47 was chosen for these experiments. Survivors of mutagen treatment were diluted and plated on to nutrient-agar plates to give $c$. 200 colonies per plate. These were replicated to nutrient-agar plates that had been spread with $0.1 \mathrm{ml}$ of a sterile filtrate of phage 
47 containing $10^{10}$ plaque-forming units per $\mathrm{ml}$. Colonies that appeared to be susceptible to lysis were examined further.

A total of 11 apparently $\mathrm{R}^{-}$mutants were isolated in five separate experiments. They all had similar properties and showed an increased sensitivity to a number of basic-set phages. The figure shows the e.o.p. of the basic-set phages on PS96 and on one of the $\mathrm{R}^{-}$mutants, $\mathrm{R}^{-}{ }_{5}$. All of the $\mathrm{R}^{-}$mutants were tested for their ability to maintain unchanged the host specificity of phage 96 and all were found to retain the property of modifying phage 96 to a form that lysed PS96 to titre, i.e., all the $\mathrm{R}^{-}$mutants were phenotypically $\mathrm{M}^{+}$. This is unlike the situation found in Escherichia coli strains K12 and B, where approximately half of single-step $\mathrm{R}^{-}$mutants are phenotypically $\mathrm{M}^{-}$(Wood, 1966) but resembles that found in the E. coli Eco RI and Eco RII systems (Yoshimori, Roulland-Dussoix and Boyer, 1972) in which restriction and modification is controlled by $R$ factors. In these latter two systems $R^{-}$ mutants are invariably $\mathrm{M}^{+}$.

Attempts were then made to isolate $\mathbf{M}^{-}$mutants from several of the $\mathrm{R}^{-}$ mutants after a second treatment with mutagens, in the following manner. After mutagenesis, the survivors were diluted and plated on to nutrient agar to give about 200 colonies per plate. Colonies were replica-plated to nutrientagar plates spread with $0 \cdot 1 \mathrm{ml}$ of a high-titre filtrate of phage 47 . All colonies on this first replica plate were somewhat sensitive to phage 47 and were obviously lytic. These lytic colonies were then replica-plated to nutrient-agar plates flooded with a broth culture of PS96. The lytic colonies on the first replica plate appeared as round zones of lysis on the lawn of PS96 on the second replica plate, since phage 47 had been modified to " 94,96 " specificity by growth in the $\mathbf{R}^{-} \mathbf{M}^{+}$colonies. It was assumed that any $\mathbf{M}^{-}$mutants present would have been detected, because these would fail to modify phage 47 and would

TABLE II

Biological properties of $S$. aureus strains in the 94, 96 complex

\begin{tabular}{ll|cc}
\hline \multicolumn{1}{c|}{ Character } & \multicolumn{2}{c}{ Number of strains } \\
& & $\overbrace{\text { tested }}^{\text {positive }}$ \\
\hline Pigmentation & & 92 & 1 \\
Lipase & & 92 & 90 \\
Fibrinolysin & & 92 & 74 \\
Haemolysins: & $\alpha$ & 31 & 28 \\
& $\beta$ & 31 & 0 \\
& $\delta$ & 31 & 22 \\
Enterotoxin: & none & 31 & 1 \\
& B & 50 & 0 \\
& C & 50 & 32 \\
& D & 50 & 0 \\
& E & 50 & 0 \\
& none & 50 & 18 \\
\hline
\end{tabular}


therefore not appear as zones of lysis on PS96. So far all of these attempts have failed. However, in the course of these experiments a second-step mutant was isolated from the $\mathrm{R}^{-}$mutant, $\mathrm{R}^{-}$, which, although apparently still $\mathrm{M}^{+}$, showed a greatly reduced ability to restrict growth of most of the basicset phages. The e.o.p. of the basic-set phages on this double mutantdesignated $\mathrm{R}^{-}{ }_{5.16}$-is shown in the figure. Twelve of the basic-set phages had an e.o.p., of 1.0 to $10^{-1}$ on the double mutant; six phages showed a marked increase in their e.o.p.; only three phages, nos. 79,84 and 85 , failed to lyse this mutant.

\section{Characteristics of strains in the 94, 96 complex}

Biological properties. Table II shows some of the characteristics of 92 of these strains. Almost all were non-pigmented and produced lipase; the majority produced both $a$ and $\delta$ haemolysins and fibrinolysin; none produced $\beta$ haemolysin. Of 50 strains examined for enterotoxin production, 32 produced enterotoxin B and 18 produced no detectable enterotoxin.

Drug resistance. One hundred and thirty-nine strains were examined for resistance to 13 antibacterial agents. Six strains were sensitive to all of the drugs: $133(95 \%)$ were penicillin-resistant, penicillinase-producers; $35 \%$ were resistant to tetracycline. However, less than $3 \%$ were resistant to any of the other antibacterial drugs against which they were tested.

\section{Discussion}

Strains in the 94, 96 complex share many properties. The majority are nonpigmented, produce lipase, fibrinolysin, $\alpha$ and $\delta$ haemolysins, enterotoxin B and penicillinase. They are generally sensitive to most antibacterial agents other than penicillin and tetracycline. They also possess a RM system that restricts the replication of phages that do not carry the " 94,96 " specificity, and modifies the DNA of phages that escape restriction to the " 94,96 " specificity.

We had little difficulty in obtaining mutants of PS96 that showed a considerable broadening of their pattern of sensitivity to lysis by the basic-set phages, and were thus presumably $\mathbf{R}^{-}$mutants. Further treatment of one of these $\left(\mathrm{R}^{-}{ }_{5}\right)$ resulted in the appearance of another mutant $\left(\mathrm{R}^{-}{ }_{5.16}\right)$ with an even wider sensitivity to lysis, but with an unimpaired ability to impose the "94, 96" specificity on any phage grown on this double mutant.

There seem to be two possible explanations for the increase in sensitivity in the double mutant in comparison with that found in the single-step mutants. One is that the presumed endonuclease, responsible for restriction in PS96, is only partially defective in the single-step mutants and continues to exert some restriction against incoming unmodified DNA, whereas the second mutation has led to a completely defective endonuclease. A second possibility is that there are two separate RM systems in PS96, one of which is mutated in the singlestep mutants and both of which are mutated in the double mutant. It is not possible at the present time to distinguish between these two possibilities. 
Failure to detect any $\mathrm{M}^{-}$mutants may be due to inadequacy of the method used to isolate them. However, if there are two separate RM systems in PS96, it may be that an $\mathrm{M}^{-}$mutation in one system would be masked by an unmutated $\mathrm{M}^{+}$gene in the second system.

The homogeneity of traits found in members of the 94, 96 complex is probably a direct consequence of the RM system they possess. Although such strains may lose some properties or acquire new ones by mutation, it is unlikely that they can acquire any new properties by gene transfer from strains outside the complex. This would have the effect of maintaining strains in the complex as a "closed community", in which transfer could occur between different members of the complex, but not between the complex and the outside world. Some confirmation of this was provided by transduction experiments. Attempts to transduce an erythromycin-resistance marker to PS96 and to the single-step mutant PS96R ${ }_{5}$ failed. However, the double mutant, PS96R ${ }^{-}{ }_{5.16}$ proved an efficient recipient and could, in turn act as donor of the transduced marker to PS96 (unpublished experiments).

RM systems have already been recognised in S. aureus (Ralston and Baer, 1964a, $b$ and $c$; van Boven et al., 1974). The best defined are those in lyticgroup-II strains. Indeed, it is the RM systems in lytic group II staphylococci that are responsible for their restricted sensitivity to the typing phages and their separation into a separate lytic group. It seems reasonable to regard strains in the 94, 96 complex as members of a new lytic group V. Their present classification as miscellaneous or unclassifiable strains is not warranted.

\section{SUMMARY}

Strains of Staphylococcus aureus that are lysed by typing phages 94 or 96, or by both phages, are usually resistant to lysis by other basic-set typing phages. They are, however, sensitive to several experimental phages and show a number of different lytic patterns when tested against these phages. These differences in susceptibility are due, in part, to immunity imposed by temperate phages carried by the different strains. Resistance to lysis by other basic-set phages was not due to prophage immunity, but to at least one restriction and modification system in such strains. Restrictionless mutants were isolated from one strain in several experiments. These showed an increased sensitivity to many basic-set phages. However, all of these mutants retained the ability to modify the phages to the characteristic " 94,96 " specificity.

Strains in the 94, 96 complex showed a remarkable homogeneity in biological traits. The majority were non-pigmented, and produced lipase, fibrinolysin, $a$ and $\delta$ haemolysins and enterotoxin B. This homogeneity may well be a reflexion of the restriction and modification systems in these strains, that prevent the acquisition of genetic material from strains outside the complex. A new lytic group $V$ is proposed for members of the 94,96 complex.

We are grateful to Dr R. J. Gilbert of Food Hygiene Laboratory, Central Public Health Laboratory, who examined our cultures for the production of enterotoxin. 


\section{REFERENCES}

Blair, J. E. and Williams, R. E. O. 1961. Phage typing of staphylococci. Bull. Wld Hlth Org., 24, 771.

Blouse, L. E., Stringfield, W. B., Marraro, R. V. and Dupuy, H. J. 1973. Activity and characteristics of a new staphylococcal phage 94. Proc. Soc. exp. Biol. Med., 142, 572.

Boven, C. P. A. van, Stobberingh, E. E., Verhoef, J. AND WinkLer, K. C. 1974. Restriction and modification of phages in staphylococcal phage typing. Ann. N.Y. Acad. Sci., 236, 376.

EleK, S. D. AND Levy, E. 1950. Distribution of haemolysins in pathogenic and nonpathogenic staphylococci. J. Path. Bact., 62, 541.

LACK, C. 1956. Biological characteristics of staphylococci recovered from pathologic materials. Ann. N.Y. Acad. Sci., 65, 103.

Novick, R. P. 1963. Analysis by transduction of mutations affecting penicillinase formation in Staphylococcus aureus. J. gen. Microbiol., 33, 121.

Ralston, D. J. AND BaER, B. S. 1964a. A new property of phage group II Staphylococcus aureus strains: host restriction of phage K14. J. gen. Microbiol., 36, 1.

Ralston, D. J. AND BaER, B. S. 1964b. Propagation of staphylococcal typing phages on a common host, Staphylococcus aureus $\mathrm{K} 1$, and host-controlled changes in their lytic range. J. gen. Microbiol., 36, 17.

RAlston, D. J. AND BAER, B. S. 1964c. Host-controlled changes in staphylococcal phage 3C affecting its broad group typing pattern. J. gen. Microbiol., 36, 25.

SIERRA, G. 1957. A simple method for the detection of lipolytic activity of micro-organisms and some observations on the influence of the contact between cells and fatty substrates. Antonie van Leeuwenhoek, 23, 15.

Šrmkovičová, M. AND GilberT, R. J. 1971. Serological detection of enterotoxin from foodpoisoning strains of Staphylococcus aureus. J. med. Microbiol., 4, 19.

SubCommitTEe on PhaGe-TYPING of StAPHYLococCI. 1975. Report (1970-74) to the International Committee on Systematic Bacteriology. Int. J. syst. Bact., 25, 241.

Willis, A. T., SMith, J. A. AND O'ConnoR, J. J. 1960. Properties of some epidemic strains of Staphylococcus aureus. J. Path. Bact., 92, 345.

Woon, W. B. 1966. Host specificity of DNA produced by Escherichia coli bacterial mutations affecting the restriction and modification of DNA. J. molec. Biol., 16, 118.

Yoshimori, R., Roulland-Dussolx, D. AND BOYER, H. W. 1972. R factor-controlled restriction and modification of deoxyribonucleic acid: restriction mutants. J. Bact., 112, 1275. 